\title{
Effect of L-ornithine-L-aspartate on patients with and without TIPS undergoing glutamine challenge: a double blind, placebo controlled trial
}

\author{
C J Rees, K Oppong, H Al Mardini, M Hudson, C O Record
}

Centre for Liver Research, University of Newcastle Upon Tyne, Newcastle Upon Tyne, UK C J Rees K Oppong

$\mathrm{H}$ Al Mardini

$M$ Hudson

C O Record

South Tyneside Health NHS Care Trust, South Tyneside, UK C J Rees

Gateshead Hospitals NHS Trust, Gateshead, UK

K Oppong

University of

Newcastle, Newcastle, UK

H Al Mardini

Newcastle Upon Tyne Hospitals NHS Trust, Newcastle Upon Tyne, UK

M Hudson

C O Record

Correspondence to: Dr C O Record, Royal Victoria Infirmary, Queen Victoria Road, Newcastle Upon Tyne NE1 4LP, UK. Email:

chrisrecord@rvincl.fsnet.co.uk

Accepted for publication 4 April 2000

\begin{abstract}
Background and aim-An oral glutamine load in cirrhotic patients awaiting liver transplantation was shown to cause a rise in blood ammonia and psychometric abnormalities which were reversed by hepatic transplantation. L-Ornithine-Laspartate (LOLA) has been shown to reduce ammonia and improve psychometric function in patients with hepatic encephalopathy. The aim of the present study was to assess the effect of LOLA in healthy patients with cirrhosis and no evidence of clinical encephalopathy after challenging the central nervous system by administration of oral glutamine.

Patients and methods-Eight cirrhotics (Child's B or C) without transjugular intrahepatic portosystemic shunts (TIPS) and seven with TIPS underwent two oral glutamine $(20 \mathrm{~g})$ challenges, receiving LOLA ( $5 \mathrm{~g}$ intravenously) on one occasion and placebo on the other in random order. Psychometric tests, including choice reaction time (CRT) and number connection test, were performed before and after glutamine, together with electroencephalography and blood ammonia.

Results-Mean basal ammonia was 27 (SEM 5) $\mu \mathrm{mol} / 1$ in non-TIPS and 76 (10) $\mu \mathrm{mol} / 1$ in TIPS patients $(p<0.05)$. Basal CRT 2 was $0.643(0.033) \mathrm{s}$ in non-TIPS and $0.825(0.076) s$ in TIPS patients $(p<0.02)$. In non-TIPS patients, ammonia increased to $36(10) \mu \mathrm{mol} / 1$ when LOLA was administered and to 62 (13) $\mu \mathrm{mol} / 1$ with placebo $(p<0.02)$. There was no alteration in psychometric function in non-TIPS patients after glutamine when LOLA was given but when placebo was given, glutamine caused prolongation of CRT $(p=0.02)$. Glutamine did not affect psychometric function in TIPS patients with or without LOLA.

Conclusion-This study showed that LOLA ameliorated the deleterious psychometric effects of glutamine in Child's grade $B$ and $C$ patients with cirrhosis without TIPS and supports its use in clinical practice in hepatic encephalopathy. (Gut 2000;47:571-574)
\end{abstract}

Keywords: hepatic encephalopathy; portosystemic encephalopathy; transjugular intrahepatic portosystemic shunt; L-ornithine-L-aspartate
Hepatic encephalopathy remains a major cause of morbidity and mortality in chronic liver disease. Gut derived toxins bypass the liver or are inadequately metabolised by the liver. The toxins then cause a complex array of neuropsychiatric disturbances. A number of toxins are implicated in the pathogenesis of encephalopathy, with ammonia remaining the main candidate neurotoxin. ${ }^{1}$

Glutamine is a non-essential neutral amino acid which is efficiently absorbed from the jejunum when administered enterally. An oral glutamine load has been shown to cause an increase in venous ammonia and associated deterioration in psychometric performance, and increase in mean amplitude of the electroencephalogram (EEG) in cirrhotic patients awaiting transplantation. ${ }^{2}$ These changes were reversed when patients were retested following orthotopic liver transplantation.

Conventional treatment of hepatic encephalopathy entails restriction of dietary protein and the use of non-absorbable disaccharides and antibiotics. L-Ornithine-L-aspartate (LOLA) is the stable salt of the amino acids ornithine and aspartic acid and has been shown to reduce blood ammonia concentration and improve psychometric performance in patients with hyperammonaemia and hepatic encephalopathy when administered intravenously. ${ }^{3-6}$ LOLA acts to stimulate the urea cycle and glutamine synthesis which are important mechanisms in ammonia detoxification. ${ }^{4-7}$

Transjugular intrahepatic portosystemic shunts (TIPS) are an established treatment in the management of variceal bleeding and in a limited number of patients with refractory ascites. $^{8}$ The incidence of hepatic encephalopathy increases in the presence of portosystemic shunts and in a recent meta-analysis, 104 of $265(39 \%)$ patients randomised to receive TIPS developed episodes of worsening or spontaneous encephalopathy compared with 54 of $267(20 \%)$ treated with endoscopic sclerotherapy with or without beta blockade. ${ }^{9}$

The aim of the present study was to evaluate the effect of LOLA on blood ammonia, psychometric function, and EEG amplitude in cirrhotic patients with and without TIPS undergoing glutamine challenge in a double blind, placebo controlled trial.

Abbreviations used in this paper: LOLA, L-ornithine-L-aspartate; TIPS, transjugular intrahepatic portosystemic shunt; CRT, choice reaction time; NCT, number connection test; EEG, electroencephalogram; CFAM, cerebral function analysing monitor. 


\section{Patients and methods}

Cirrhotic patients with and without TIPS were invited to participate in the study and written informed consent was obtained. TIPS patients were recruited at the time of routine portal venography designed to test the patency and pressure gradient across the shunt, which had been inserted 3-24 months before the test. Some patients required balloon dilatation of the shunt to decrease the pressure gradient to less than $12 \mathrm{~mm} \mathrm{Hg}$ but no sedation was given. Following the procedure the portal catheter was left in situ for 24 hours to allow portal venous sampling on two consecutive mornings. Non-TIPS patients with cirrhosis attended on two separate days on an outpatient basis at least one week apart.

Patients underwent psychometric and EEG assessment before and after glutamine challenge. Psychometric testing was performed in a quiet environment by a single investigator (CJR). A standard trailmaking test was performed consisting of 25 circles joined up sequentially by the patient. The trailmaking test serves as a measure of visual conceptual and visuomotor tracking. Patients' choice reaction time (CRT) to light was tested using the Leeds psychomotor tester. CRT measures the speed of a patient's motor response to a choice of visual stimuli. ${ }^{2}$ The patient is presented with an array of six lamps which light up in random order. Choice reaction time 1 (CRT1) or lift off time is the interval between presentation of the visual stimulus and recognition (measured by the patient lifting off his finger from the start button). CRT2 is the total time between presentation of the visual signal and cancellation of the signal by pressing the appropriate touch sensitive button next to the light. Analysed EEG recording was performed using a cerebral function analysing monitor (CFAM). ${ }^{2}$ This provides a continuous detailed analysis of amplitude trends and frequency distribution of the weighted EEG signal derived from a single pair of surface electrodes. Data from the CFAM machine were stored in a data recorder and downloaded onto a PC for statistical analysis.

All studies were performed in the morning following an overnight fast. Patients were randomised double blind (by allocation to the next numbered supply of medication) to receive an intravenous LOLA infusion or placebo. LOLA (5 g) or placebo was diluted to $50 \mathrm{ml}$ with $\mathrm{N}$-saline and infused over one hour commencing at time zero. A basal number connection test (NCT), CRT to light, and CFAM recording were performed before the

Table 1 Baseline ammonia levels, choice reaction time 1 and 2 (CRT1 and CRT2), number connection test (NCT), and mean EEG amplitude in TIPS and non-TIPS patients (mean (SEM))

\begin{tabular}{lccc}
\hline & $\begin{array}{l}\text { Non-TIPS } \\
(n=8)\end{array}$ & $\begin{array}{l}\text { TIPS } \\
(n=7)\end{array}$ & p Value \\
\hline Ammonia $(\mu / 1)$ & $27(5)$ & $76(10)$ & $<0.05$ \\
CRT1 $(\mathrm{ms})$ & $412(20)$ & $506(53)$ & 0.12 \\
CRT2 $(\mathrm{ms})$ & $643(33)$ & $825(76)$ & $<0.02$ \\
NCT $(\mathrm{s})$ & $43(6)$ & $52(7)$ & 0.46 \\
EEG amplitude $(\mu \mathrm{V})$ & $72(3)$ & $72(3)$ & 1.0 \\
\hline
\end{tabular}

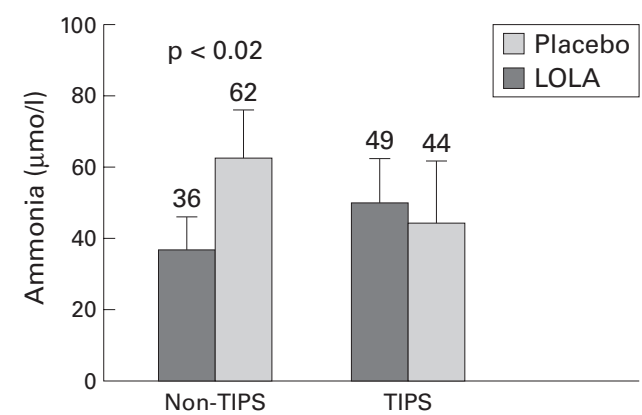

Figure 1 Increase in venous ammonia following glutamine challenge in TIPS and non-TIPS patients after administration of L-ornithine-L-aspartate (LOLA) or placebo (mean (SEM)).

infusion was commenced. Half way through the infusion at time 30 minutes, patients were given $20 \mathrm{~g}$ of oral glutamine (3.8 $\mathrm{g}$ of nitrogen; equivalent to $24 \mathrm{~g}$ of protein). Peripheral venous sampling was performed at times 0,30 , 60,90 , and 120 minutes to measure whole blood ammonia concentration (Ammonia Checker II, Model AA-4120; Kyoto Daüchi Kagaku Co Ltd). ${ }^{10}$ In TIPS patients, portal blood sampling was also undertaken. At the end of 120 minutes the psychometric testing and CFAM recording were repeated (a different format of the NCT to basal was used).

The study was approved by the Joint Ethics Committee of Newcastle and North Tyneside Health Authority, University of Newcastle, and University of Northumbria at Newcastle.

Data are presented as mean (SEM) or median (range). Differences were examined using the Mann-Whitney $U$ test (unpaired data) or the Wilcoxon signed rank test (paired data).

\section{Results}

Eight patients without TIPS and seven patients with TIPS were recruited into the study. Mean age was 50 (3.8) years in non-TIPS and 54 (3.6) years in TIPS patients. The aetiology of the cirrhosis was alcohol in all eight non-TIPS patients and in six of seven TIPS patients, with cryptogenic cirrhosis accounting for the remaining patient. All patients had stable liver function and none was abusing alcohol or consuming alcohol or psychoactive drugs at the time of the test or in the period between recruitment and the test. The non-TIPS cirrhotics included seven patients with Child

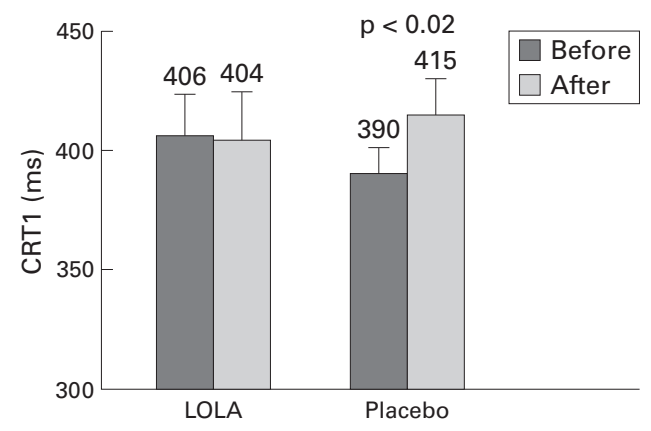

Figure 2 Change in choice reaction time 1 (CRT1) in non-TIPS patients before and after glutamine challenge in the L-ornithine-L-aspartate (LOLA) and placebo groups (mean (SEM)). 
Table 2 Choice reaction time 2 (CRT2), number connection test (NCT), and mean EEG amplitude in non-TIPS patients before and after glutamine in the L-ornithine-L-aspartate (LOLA) and placebo groups (mean (SEM); $n=8$ )

\begin{tabular}{llllll}
\hline & \multicolumn{2}{l}{$L O L A$} & & \multicolumn{2}{l}{ Placebo } \\
\cline { 2 - 3 } \cline { 5 - 6 } & Pre-glutamine & Post-glutamine & & Pre-glutamine & Post-glutamine \\
\hline CRT2 $(\mathrm{ms})$ & $652(33)$ & $651(24)$ & & $627(23)$ & $649(24)$ \\
NCT $(\mathrm{s})$ & $39(5)$ & $35(3)$ & & $37(6)$ & $40(8)$ \\
EEG amplitude $(\mu \mathrm{V})$ & $71(3)$ & $73(3)$ & & $67(3)$ & $69(4)$ \\
\hline
\end{tabular}

Table 3 Choice reaction time 1 and 2 (CRT1 and CRT2), number connection test (NCT), and EEG amplitude in TIPS patients before and after glutamine challenge in the L-ornithine-L-aspartate (LOLA) and placebo groups (mean (SEM); $n=7$ )

\begin{tabular}{llllll}
\hline & \multicolumn{2}{l}{ LOLA } & & & \multicolumn{2}{l}{ Placebo } \\
\cline { 2 - 3 } \cline { 5 - 6 } \cline { 5 - 6 } & Pre-glutamine & Post-glutamine & & Pre-glutamine & Post-glutamine \\
\hline CRT1 (ms) & $472(31)$ & $473(34)$ & & $512(61)$ & $477(34)$ \\
CRT2 (ms) & $820(57)$ & $808(70)$ & & $822(88)$ & $797(54)$ \\
NCT $(\mathrm{s})$ & $52(6)$ & $57(7)$ & & $58(8)$ & $63(8)$ \\
EEG amplitude $(\mu \mathrm{V})$ & $69(4)$ & $72(4)$ & & $75(5)$ & $70(3)$ \\
\hline
\end{tabular}

Pugh grade B liver disease and one with grade C disease. Among TIPS patients, five had grade $\mathrm{B}$ and two grade $\mathrm{C}$ disease. No patient was receiving chronic medication for prophylaxis of encephalopathy.

Table 1 shows comparisons of baseline values in TIPS and non-TIPS patients. Basal ammonia and CRT2 were greater in TIPS patients.

Glutamine challenge produced an increase in ammonia in all patients with a mean increase of 48 (7) $\mu \mathrm{mol} / 1$. The peak value occurred at 60 or 90 minutes.

Figure 1 demonstrates the rise in venous ammonia in TIPS and non-TIPS patients when LOLA and placebo were administered. In non-TIPS patients, the increase in ammonia was significantly attenuated by administration of LOLA compared with placebo. In TIPS patients, no difference between LOLA and placebo was observed.

Basal portal venous ammonia levels in TIPS patients were $62-225 \mu \mathrm{mol} / 1$ with a mean rise of 121 (45-329) $\mu \mathrm{mol} / 1$ following glutamine challenge. No difference was demonstrated in the portal ammonia rise between LOLA and placebo administration.

In non-TIPS patients, glutamine caused prolongation of CRT1 when placebo was administered (390 (12) ms before challenge, 415 (16) ms after challenge) but not when LOLA was administered (406 (19) ms before challenge, 404 (21) ms after challenge) $(p<0.02)$ (fig 2). Among these non-TIPS patients, no difference in effect was demonstrated between LOLA and placebo on CRT2, NCT, or mean EEG amplitude (table 2).

In TIPS patients, glutamine challenge did not have any demonstrable effect on psychometric function or mean EEG amplitude. No differences were found between LOLA and placebo in these patients (table 3).

\section{Discussion}

Therapeutic options in the management of hepatic encephalopathy are disappointing. Current treatment aims to reduce absorption of neurotoxins from the gastrointestinal tract, and low protein diet, non-absorbable disaccha- ride (lactulose and lactitol), and gut sterilising antibiotics are measures currently used. ${ }^{11} \mathrm{~A}$ wide range of other treatments have been studied. These include administration of branched chain amino acids, levodopa, bromocriptine, and flumazenil. ${ }^{11}$

Previous controlled studies ${ }^{4}{ }^{5}$ have demonstrated the effect of intravenous LOLA on ammonia concentration and mental function in patients with advanced liver disease but without portosystemic shunts. HengleinOttermann ${ }^{3}$ demonstrated that compared with placebo, LOLA lowered ammonia concentration following ammonium chloride infusion. In a double blind, placebo controlled, crossover, randomised controlled trial, ${ }^{4}$ three dosages of intravenous LOLA $(5,20$, and $40 \mathrm{~g}$ per day) were compared with placebo (saline) in 10 cirrhotic patients with mild hepatic encephalopathy (grade $<2$ ) and hyperammonaemia $(>70 \mu \mathrm{mol} / \mathrm{l})$. Hyperammonaemia was induced by oral ingestion of a liquid protein diet which contained $0.75 \mathrm{~g}$ of protein per $\mathrm{kg}$, given in two divided doses $(0.25 \mathrm{~g} / \mathrm{kg}$ and $0.50 \mathrm{~g} / \mathrm{kg}$ given four hours apart). A postprandial elevation in blood ammonia concentration was observed after each ingestion of protein. Infusion of LOLA reduced venous ammonia levels in a dose related manner whereas placebo had no such effect. Blood ammonia concentrations did not increase throughout the $40 \mathrm{~g}$ infusion of LOLA. A randomised controlled study of 126 cirrhotic patients with hyperammonaemia and hepatic encephalopathy has recently been published. ${ }^{5}$ Infusion of LOLA lowered ammonia levels and improved psychometric performance in patients with both overt and subclinical encephalopathy. LOLA was well tolerated with only three patients developing minor adverse events. In a further study, ${ }^{6}$ LOLA was associated with significant improvements in NCT, mental state grade, and portosystemic encephalopathy index compared with placebo in patients with chronic hepatic encephalopathy.

In the current study in non-encephalopathic patients, glutamine challenge caused an increase in venous ammonia in non-TIPS patients which was accompanied by deterioration in CRT to light. However, this was not the case in TIPS patients. TIPS patients had significantly higher baseline ammonia levels, and baseline psychometric function (as shown by CRT2) was significantly worse than in nonTIPS cirrhotics. These data suggest that while TIPS patients did not have overt hepatic encephalopathy, subclinical encephalopathy (abnormal psychometry) was present.

In the present study intravenous LOLA ameliorated the rise in ammonia and change in CRT1 following glutamine in cirrhotic patients without TIPS. In cirrhotic patients with TIPS, no alteration in psychometric function was demonstrated between LOLA and placebo. LOLA may have been ineffective in TIPS patients because of enhanced portosystemic shunting and a reduction in hepatic sinusoidal blood flow in these patients, as shown by a significant decrease in indocyanine green clearance after the procedure. ${ }^{12}$ The test was undertaken shortly after portal venography when the 
shunt was dilated to achieve portal decompression below a wedged hepatic venous gradient of $12 \mathrm{~mm} \mathrm{Hg}$ so that at the time of the test portosystemic shunting was maximised. Thus the fall in hepatic sinusoidal blood flow would effectively remove the drug from its site of action within the liver parenchyma. A higher dose of the drug may be required in such patients. It has recently been shown that the prevalence of hepatic encephalopathy decreases in TIPS patients three months or more after the procedure despite a sustained elevation in blood ammonia concentration. This was attributed to cerebral adaptation to gut derived neurotoxins ${ }^{13}$ and this effect could explain our inability to demonstrate psychometric improvement with LOLA.

Previous studies of LOLA ${ }^{3-6}$ have demonstrated the ability of this substance to lower blood ammonia and to improve psychometric function in patients with overt and subclinical hepatic encephalopathy. We used the previously described glutamine challenge ${ }^{2}$ as a controlled, reproducible, clinical model for studying hepatic encephalopathy. Under these conditions there are no other precipitating or exacerbating clinical factors which might influence trial outcome. Using this model, LOLA infusions were effective in reducing the increase in ammonia and deterioration in psychometric function following glutamine challenge in cirrhotic patients without prosthetic portosystemic shunts and this result supports its use in clinical practice. The beneficial effect of LOLA is consistent with ammonia playing a role in the pathogenesis of hepatic encephalopathy.

This work was undertaken with the aid of grants from Merz and Co (60318 Frankfurt/Main, Germany) who also kindly supplied
L-ornithine-L-aspartate and matching placebo, and Dame Catherine Cookson. Dr P Senior kindly assisted with the statistical analysis.

1 Record CO. Neurochemistry of hepatic encephalopathy. Gut 1991;32:1261-3.

2 Oppong $\mathrm{K}$, Al-Mardini $\mathrm{H}$, Thick $\mathrm{M}$, et al. Oral glutamine challenge in cirrhotics awaiting liver transplantation: A psychometric and analysed EEG study. Hepatology 1997;26: $870-6$.

3 Henglein-Ottermann D. Der EinfluB von Ornithin-Aspartat auf die experimentell erzeugte Hyperammoniame. Klinischexperimentelle Studie. Ther Gegenw 1976;115:150418.

4 Staedt U, Leweling H, Gladisch R, et al. Effects of ornithine aspartate on plasma ammonia and plasma amino acid in patients with cirrhosis. A double-blind, randomised study patients with cirrhosis. A double-blind, randomised study 30 .

5 Kircheis G, Nilius R, Held C, et al. Therapeutic efficacy of 1-ornithine-1-aspartate infusions in patients with cirrhosis and hepatic encephalopathy: results of a placebocontrolled, double-blind study. Hepatology 1997;25:135160.

6 Rose C, Michalak A, Pannunzio P, et al. Ornithine L aspartate in experimental portalsystemic encephalopathy: therapeutic efficacy and mechanism of action. Metab Brain Dis 1998;13:147-57.

7 Stauch S, Kircheis G, Adler G, et al. Oral L-ornithine-Laspartate therapy of chronic hepatic encephalopathy: results of a placebo-controlled double-blind study. 7 results of a placebo-contr.
Hepatol 1998;28:856-64.

8 Rees CJ, Hudson M, Record CO. Therapeutic modalities in portal hypertension. Eur f Gastroenterol Hepatol 1997;9:911.

9 Record CO, Al Mardini H, MacRae K. TIPS or endoscopic \pm pharmacological treatment for bleeding varices: A meta analysis. Hepatology 1998;28:662A.

10 Huizenga JR, Tangerman A, Gips CH. A rapid method for blood ammonia determination using the new blood ammonia checker (BAC) II. Clin Chim Acta 1992:210:153-5.

11 Jalan R, Seery JP, Taylor-Robinson SD. Pathogenesis and treatment of chronic hepatic encephalopathy. Aliment Pharmacol Ther 1996;10:681-97.

12 Haag K, Lu S, Rossle M, et al. The influence of TIPS on portal haemodynamics and liver function with respect to hepatic encephalopathy and survival. Hepatology 1995;22: 296A.

13 Nolte W, Wiltfang J, Schindler C, et al. Portosystemic hepatic encephalopathy after transjugular intrahepatic portosystemic shunt in patients with cirrhosis: clinical, laboratory, psychometric, and electroencephalographic investigations. Hepatology 1998;28:1215-25. 